\title{
Singular value inequalities with applications
}

\section{Wasim Audeh}

Department of Mathematics, University of Petra, Amman, Jordan.

\section{Abstract}

Let $A_{i}, B_{i}, X_{i}, Y_{i}$ be $n \times n$ complex matrices, $i=1,2, \ldots, m$ and let $f$ be a nonnegative increasing convex function on an interval I such that $0 \in I$ and $f(0) \leqslant 0$. Then

$$
2 s_{j}\left(f\left(\left|\sum_{i=1}^{m} A_{i} X_{i} Y_{i}^{*} B_{i}^{*}\right|\right)\right) \leqslant(\max \{S, T\})^{2} s_{j}(K)
$$

for $j=1,2, \ldots, n$, where

$$
\begin{aligned}
S & =\left\|\sum_{i=1}^{m} A_{i} A_{i}^{*}\right\|^{1 / 2}, T=\left\|\sum_{i=1}^{m} B_{i} B_{i}^{*}\right\|^{1 / 2}, \\
K & =f\left(\left|X_{1}\right|^{2}+\left|Y_{1}\right|^{2}\right) \oplus \ldots \oplus f\left(\left|X_{m}\right|^{2}+\left|Y_{m}\right|^{2}\right)
\end{aligned}
$$

and $\max \{S, T\} \leqslant 1$. Several singular value inequalities are also proved.

Keywords: Singular value, convex function, positive operator, inequality.

2020 MSC: 15A18, 15A42, 15A60, 47A63, 47B05, 47B15.

(C)2022 All rights reserved.

\section{Introduction}

Let $\mathbb{M}_{n}(\mathbb{C})$ denote the algebra of all $n \times n$ complex matrices. For $A \in \mathbb{M}_{n}(\mathbb{C})$, the singular values of $A$ are denoted by $s_{1}(A) \geqslant s_{2}(A) \geqslant \ldots \geqslant s_{n}(A)$, they are precisely the eigenvalues of the positive matrix $|A|=\left(A^{*} A\right)^{1 / 2}$, Singular values have several properties: Let $A, B \in \mathbb{M}_{\mathfrak{n}}(\mathbb{C})$. Then

(a) $s_{j}(A)=s_{j}\left(A^{*}\right)=s_{j}(|A|)$ for $j=1,2, \ldots, n$.

(b) $s_{j}\left(A A^{*}\right)=s_{j}\left(A^{*} A\right)$ for $j=1,2, \ldots, n$.

(c) $s_{j}(A) \leqslant s_{j}(B)$ if and only if $s_{j}(A \oplus A) \leqslant s_{j}(B \oplus B)$ for $j=1,2, \ldots, n$.

(d) $s_{j}\left[\begin{array}{cc}A & 0 \\ 0 & B\end{array}\right]=s_{j}\left[\begin{array}{cc}0 & B \\ A & 0\end{array}\right]$ for $j=1,2, \ldots, n$, where the singular values of $\left[\begin{array}{cc}A & 0 \\ 0 & B\end{array}\right]$ consist of these of $A$ together with those of $B$.

Email address: waudeh@uop.edu.jo (Wasim Audeh)

doi: $10.22436 /$ jmcs.024.04.04

Received: 2021-01-21 Revised: 2021-02-18 Accepted: 2021-03-18 
(Extensive studies on singular values are available in $[1,8,12])$.

Bhatia and Kittaneh in [9] proved the A.G.M.I. for singular values: If $A, B \in \mathbb{M}_{n}(\mathbb{C})$, then

$$
2 s_{j}\left(A B^{*}\right) \leqslant s_{j}\left(A^{*} A+B^{*} B\right)
$$

for $j=1,2, \ldots, n$. Hirzallah in [11] gave a generalization of inequality (1.1): If $A_{i} \in \mathbb{B}(\mathbb{H}), i=1,2,3,4$, then

$$
\sqrt{2} s_{j}\left(\left|A_{1} A_{2}^{*}+A_{3} A_{4}^{*}\right|^{1 / 2}\right) \leqslant s_{j}\left(\left[\begin{array}{ll}
A_{1} & A_{3} \\
A_{2} & A_{4}
\end{array}\right]\right)
$$

for $j=1,2, \ldots, 2 n$. Audeh in [3] created a generalization of inequality (1.2): Let $A_{i}, B_{i}, X_{i}, Y_{i} \in \mathbb{M}_{n}(\mathbb{C})$ such that $X_{i}$ and $Y_{i}$ are positive, $i=1,2, \ldots, n$. Then

$$
2 s_{j}\left(\sum_{i=1}^{n} A_{i} X_{i}^{1 / 2} Y_{i}^{1 / 2} B_{i}^{*}\right) \leqslant s_{j}^{2}(W)
$$

for $j=1,2, \ldots, n$, where $W=\left[\begin{array}{cccc}A_{1} X_{1}^{1 / 2} & A_{2} X_{2}^{1 / 2} & \ldots & A_{n} X_{n}^{1 / 2} \\ B_{1} Y_{1}^{1 / 2} & B_{2} Y_{2}^{1 / 2} & \ldots & B_{n} Y_{n}^{1 / 2}\end{array}\right]$. This generalization contains two attractive special cases. The first one: Let $A, B, X \in \mathbb{B}(\mathbb{H})$ such that $X$ is positive. Then

$$
2 s_{j}\left(A X^{1 / 2} Y^{1 / 2} B^{*}+B X^{1 / 2} Y^{1 / 2} A^{*}\right) \leqslant s_{j}^{2}\left(\left[\begin{array}{cc}
A X^{1 / 2} & B X^{1 / 2} \\
B Y^{1 / 2} & A Y^{1 / 2}
\end{array}\right]\right)
$$

for $j=1,2, \ldots, n$. The second one: Let $A, B, X \in \mathbb{B}(\mathbb{H})$ be positive. Then

$$
2 s_{j}(M+N) \leqslant s_{j}\left(\left(H+\left|K^{*}\right|\right) \oplus(L+|K|)\right.
$$

for $j=1,2, \ldots, n$, where $M=A^{1 / 2} X^{1 / 2} Y^{1 / 2} A^{1 / 2}, N=B^{1 / 2} X^{1 / 2} Y^{1 / 2} B^{1 / 2}, H=X^{1 / 2} A X^{1 / 2}+Y^{1 / 2} A Y^{1 / 2}$, $\mathrm{K}=\mathrm{X}^{1 / 2} \mathrm{~A}^{1 / 2} \mathrm{~B}^{1 / 2} \mathrm{X}^{1 / 2}+\mathrm{Y}^{1 / 2} \mathrm{~A}^{1 / 2} \mathrm{~B}^{1 / 2} Y^{1 / 2}$ and $\mathrm{L}=\mathrm{X}^{1 / 2} \mathrm{~B} X^{1 / 2}+\mathrm{Y}^{1 / 2} \mathrm{~B} \mathrm{Y}^{1 / 2}$.

In this paper, we provide a new general inequality which is a generalization of several inequalities, one of these inequalities is inequality (1.1). Moreover, we compare our findings with inequalities (1.3) and (1.4). For recent studies on this topic, the reader should return to [2-6].

\section{Main results}

The following lemmas are essential for supporting our conclusions. The first lemma follows from the min-max principle (see, e.g., [1, p. 75] or [9, p. 27]). The second and third lemmas are shown in [7]. The fourth lemma is proved in [3].

Lemma 2.1. Let $A, B, X \in \mathbb{M}_{\mathfrak{n}}(\mathbb{C})$. Then

$$
s_{j}(A X B) \leqslant\|A\|\|B\| s_{j}(X)
$$

for $j=1,2, \ldots, n$.

Lemma 2.2. Let $A \in \mathbb{M}_{n}(\mathbb{C})$ and let $f \geqslant 0$ and increasing function on an interval $I$. Then, for $j=1,2, \ldots, n$,

$$
f\left(s_{\mathfrak{j}}(A)\right)=s_{\mathfrak{j}}(f(|A|)) .
$$

2. For Hermitian $A$,

$$
f\left(\lambda_{j}(A)\right)=\lambda_{j}(f(A)) .
$$


Lemma 2.3. Let $\mathrm{A}, \mathrm{X} \in \mathrm{M}_{\mathfrak{n}}(\mathbb{C})$ such that $\mathrm{A}$ is Hermitian and $\mathrm{X}$ is contraction and let $\mathrm{f}$ be a nonnegative monotone convex function on an interval $I$ such that $0 \in \mathrm{I}$ and $\mathrm{f}(0) \leqslant 0$. Then

$$
\lambda_{j}\left(f\left(X^{*} A X\right)\right) \leqslant \lambda_{j}\left(X^{*} f(A) X\right)
$$

for $j=1,2, \ldots, n$.

Lemma 2.4. Let $A, B, X, Y \in \mathbb{M}_{n}(\mathbb{C})$. Then

$$
s_{j}\left(A X Y^{*} B^{*}\right) \leqslant \frac{1}{2} s_{j}\left(X^{*}|A|^{2} X+Y^{*}|B|^{2} Y\right)
$$

for $j=1,2, \ldots, n$.

Now we are ready to state the first result of this paper, all functions considered in our results are nonnegative increasing convex functions on the interval I such that $0 \in \operatorname{I}$ and $f(0) \leqslant 0$.

Theorem 2.5. Let $A, B, X, Y \in \mathbb{M}_{n}(\mathbb{C})$ such that $\max \{\|A\|,\|B\|\} \leqslant 1$. Then

$$
2 s_{j}\left(f\left(\left|A X Y^{*} B^{*}\right|\right)\right) \leqslant(\max \{\|A\|,\|B\|\})^{2} s_{j}\left(f\left(X^{*} X+Y^{*} Y\right)\right)
$$

for $j=1,2, \ldots, n$.

Proof. Throughout this proof, let $Z=\left[\begin{array}{ll}X & 0 \\ Y & 0\end{array}\right]$, then

$$
\begin{aligned}
2 s_{j}\left(f\left(\left|A X Y^{*} B^{*}\right|\right)\right. & =2 f\left(s_{j}\left(A X Y^{*} B^{*}\right)\right) \quad(\text { by Lemma 2.2) } \\
& \leqslant f\left(s_{j}\left(X^{*}|A|^{2} X+Y^{*}|B|^{2} Y\right)\right)
\end{aligned}
$$

(by Lemma 2.4 and since $f$ is increasing function)

$$
\begin{aligned}
& =f\left(s_{j}\left(Z^{*}\left[\begin{array}{cc}
|A|^{2} & 0 \\
0 & |B|^{2}
\end{array}\right] Z\right)\right) \\
& =f\left(\lambda_{j}\left(Z^{*}\left[\begin{array}{cc}
|A|^{2} & 0 \\
0 & |B|^{2}
\end{array}\right] Z\right)\right) \\
& =f\left(\lambda_{j}\left(\left[\begin{array}{cc}
|A| & 0 \\
0 & |B|
\end{array}\right] Z Z^{*}\left[\begin{array}{cc}
|A| & 0 \\
0 & |B|
\end{array}\right]\right)\right) \\
& =\lambda_{j}\left(f\left(\left[\begin{array}{cc}
|A| & 0 \\
0 & |B|
\end{array}\right] Z Z^{*}\left[\begin{array}{cc}
|A| & 0 \\
0 & |B|
\end{array}\right]\right)\right) \\
& \leqslant \lambda_{j}\left(\left[\begin{array}{cc}
|A| & 0 \\
0 & |B|
\end{array}\right] f\left(Z Z^{*}\right)\left[\begin{array}{cc}
|A| & 0 \\
0 & |B|
\end{array}\right]\right)
\end{aligned}
$$

(by Lemma 2.3)

$$
\begin{aligned}
& =s_{j}\left(\left[\begin{array}{cc}
|A| & 0 \\
0 & |B|
\end{array}\right] f\left(Z Z^{*}\right)\left[\begin{array}{cc}
|A| & 0 \\
0 & |B|
\end{array}\right]\right) \\
& \leqslant\left\|\left[\begin{array}{cc}
|A| & 0 \\
0 & |B|
\end{array}\right]\right\|^{2} s_{j}\left(f\left(Z Z^{*}\right)\right)
\end{aligned}
$$

(by Lemma 2.1)

$$
=\left\|\left[\begin{array}{cc}
|A| & 0 \\
0 & |B|
\end{array}\right]\right\|^{2} f\left(s_{j}\left(Z Z^{*}\right)\right)
$$

(by Lemma 2.2) 


$$
\begin{aligned}
& =\left\|\left[\begin{array}{cc}
|A| & 0 \\
0 & |B|
\end{array}\right]\right\|^{2} f\left(s_{j}\left(Z^{*} Z\right)\right) \\
& =(\max \{\|A\|,\|B\|\})^{2} s_{j}\left(f\left(X^{*} X+Y^{*} Y\right)\right) .
\end{aligned}
$$

Corollary 2.6. Let $\mathrm{A}, \mathrm{B}, \mathrm{X}, \mathrm{Y} \in \mathbb{B}(\mathbb{H})$ such that $\max \{\|\mathrm{A}\|,\|\mathrm{B}\|\} \leqslant 1, \mathrm{X}$ and $\mathrm{Y}$ are positive. Then

$$
2 s_{j}\left(f\left(\left|A X^{1 / 2} Y^{1 / 2} B^{*}\right|\right) \leqslant(\max \{\|A\|,\|B\|\})^{2} s_{j}(f(X+Y))\right.
$$

for $\mathrm{j}=1,2, \ldots, \mathrm{n}$.

Proof. Substituting $X$ by $X^{1 / 2}$ and $Y$ by $Y^{1 / 2}$ in inequality (2.3), we give inequality (2.4).

Corollary 2.7. Let $A, B, X, Y \in \mathbb{M}_{n}(\mathbb{C})$ be positive such that $\max \{\|A\|,\|B\|\} \leqslant 1$. Then

$$
2 s_{j}\left(f\left(\left|A^{1 / 2} X^{1 / 2} Y^{1 / 2} B^{1 / 2}\right|\right) \leqslant\left(\max \left\{\|A\|^{1 / 2},\|B\|^{1 / 2}\right\}\right)^{2} s_{j}(f(X+Y))\right.
$$

for $\mathrm{j}=1,2, \ldots, \mathrm{n}$.

Proof. The result is deduced from inequality (2.4) by letting $A=A^{1 / 2}$ and $B=B^{1 / 2}$.

The following is our main result.

Theorem 2.8. Let $A_{i}, B_{i}, X_{i}, Y_{i} \in \mathbb{M}_{n}(\mathbb{C}), i=1,2, \ldots, m$. Then

$$
2 s_{j}\left(f\left(\left|\sum_{i=1}^{m} A_{i} X_{i} Y_{i}^{*} B_{i}^{*}\right|\right)\right) \leqslant(\max \{S, T\})^{2} s_{j}(K)
$$

for $j=1,2, \ldots, n$, where

$$
\begin{aligned}
& S=\left\|\sum_{i=1}^{m} A_{i} A_{i}^{*}\right\|^{1 / 2}, \quad T=\left\|\sum_{i=1}^{m} B_{i} B_{i}^{*}\right\|^{1 / 2}, \\
& K=f\left(\left|X_{1}\right|^{2}+\left|Y_{1}\right|^{2}\right) \oplus \ldots \oplus f\left(\left|X_{m}\right|^{2}+\left|Y_{m}\right|^{2}\right)
\end{aligned}
$$

and $\max \{\mathrm{S}, \mathrm{T}\} \leqslant 1$.

Proof. On $\oplus_{j=1}^{n} \mathrm{H}$, Let

$$
\begin{aligned}
& A=\left[\begin{array}{cccc}
A_{1} & A_{2} & \ldots & A_{n} \\
0 & 0 & \cdots & 0 \\
\vdots & \vdots & \ddots & \vdots \\
0 & 0 & \cdots & 0
\end{array}\right], \quad B=\left[\begin{array}{cccc}
B_{1} & B_{2} & \ldots & B_{n} \\
0 & 0 & \cdots & 0 \\
\vdots & \vdots & \ddots & \vdots \\
0 & 0 & \cdots & 0
\end{array}\right], \\
& X=\left[\begin{array}{cccc}
X_{1} & 0 & \ldots & 0 \\
0 & X_{2} & \cdots & 0 \\
\vdots & \vdots & \ddots & \vdots \\
0 & 0 & \cdots & X_{n}
\end{array}\right], \text { and } Y=\left[\begin{array}{cccc}
Y_{1} & 0 & \ldots & 0 \\
0 & Y_{2} & \cdots & 0 \\
\vdots & \vdots & \ddots & \vdots \\
0 & 0 & \cdots & Y_{n}
\end{array}\right] .
\end{aligned}
$$

Then

$$
A X Y^{*} B^{*}=\left[\begin{array}{cccc}
\sum_{i=1}^{n} A_{i} X_{i} Y_{i}^{*} B_{i}^{*} & 0 & \ldots & 0 \\
0 & 0 & \cdots & 0 \\
\vdots & \vdots & \ddots & \vdots \\
0 & 0 & \cdots & 0
\end{array}\right] \text {, }
$$




$$
\begin{gathered}
\|A\|=\left\|\sum_{i=1}^{m} A_{i} A_{i}^{*}\right\|^{1 / 2},\|B\|=\left\|\sum_{i=1}^{m} B_{i} B_{i}^{*}\right\|^{1 / 2} \text {, and } \\
s_{j}\left(f\left(X^{*} X+Y^{*} Y\right)\right)=s_{j}(K) .
\end{gathered}
$$

Apply inequality (2.5) to the operator matrices $A, B, X$ and $Y$, we give inequality (2.6).

Corollary 2.9. Let $A_{i}, B_{i}, X_{i}, Y_{i} \in \mathbb{M}_{n}(\mathbb{C}), i=1,2$ such that

$$
\max \left\{\left\|A_{1} A_{1}^{*}+A_{2} A_{2}^{*}\right\|^{1 / 2},\left\|B_{1} B_{1}^{*}+B_{2} B_{2}^{*}\right\|^{1 / 2}\right\} \leqslant 1 .
$$

Then

$$
2 s_{j}\left(f\left(\left|\sum_{i=1}^{2} A_{i} X_{i} Y_{i}^{*} B_{i}^{*}\right|\right)\right) \leqslant(\max \{P, Q\})^{2} s_{j}(M \oplus N)
$$

for $j=1,2, \ldots, n$, where

$$
\begin{aligned}
P & =\left\|A_{1} A_{1}^{*}+A_{2} A_{2}^{*}\right\|^{1 / 2}, \\
Q & =\left\|B_{1} B_{1}^{*}+B_{2} B_{2}^{*}\right\|^{1 / 2}, \\
M & =f\left(\left|X_{1}\right|^{2}+\left|Y_{1}\right|^{2}\right)
\end{aligned}
$$

and

$$
N=f\left(\left|X_{2}\right|^{2}+\left|Y_{2}\right|^{2}\right)
$$

Proof. Letting $A_{i}=B_{i}=X_{i}=Y_{i}=0$ for $i=3,4, \ldots, n$ in inequality (2.6), we give inequality (2.7).

Remark 2.10. Let $A_{2}=B_{2}=X_{2}=Y_{2}=0, A_{1}=B_{1}=I$ and let $f(t)=t$ in inequality (2.7), we give inequality (1.1).

We will present some applications.

Corollary 2.11. Let $A, B, X, Y \in \mathbb{M}_{n}(\mathbb{C}), i=1,2$ such that $X_{i}, Y_{i} \geqslant 0$ and $N=((X+Y) \oplus(X+Y))$. Then

$$
2 s_{j}\left(A X^{1 / 2} Y^{1 / 2} B^{*}+B X^{1 / 2} Y^{1 / 2} A^{*}\right) \leqslant\left\|A A^{*}+B B^{*}\right\|^{1 / 2} s_{j}(N)
$$

for $j=1,2, \ldots, n$.

Proof. Substituting $A_{1}=B_{2}=A, A_{2}=B_{1}=B, X_{1}=X_{2}=X^{1 / 2}, Y_{1}=Y_{2}=Y^{1 / 2}$ and $f(t)=t$ in inequality (2.7), we get our result.

Remark 2.12. You can easily prove that inequality (2.8) is sharper than inequality (1.3) when $A=B=I$. To see this, note that

$$
\sqrt{2} s_{j}((X+Y) \oplus(X+Y)) \leqslant 2 s_{j}((X+Y) \oplus(X+Y)) .
$$

Remark 2.13. Substitute $Y=X$ in inequality (2.8), we obtain

$$
s_{j}\left(A X B^{*}+B X A^{*}\right) \leqslant\left\|A A^{*}+B B^{*}\right\|^{1 / 2} s_{j}(X \oplus X)
$$

for $j=1,2, \ldots, n$.

Remark 2.14. Substitute $\mathrm{X}=\mathrm{Y}=\mathrm{I}$ in inequality (2.9), we obtain

$$
s_{j}\left(A B^{*}+B A^{*}\right) \leqslant\left\|A A^{*}+B B^{*}\right\|^{1 / 2}
$$

for $j=1,2, \ldots, n$. In particular,

$$
\left\|A B^{*}+B A^{*}\right\| \leqslant\left\|A A^{*}+B B^{*}\right\|^{1 / 2} .
$$


Corollary 2.15. Let $A, B, X, Y \in \mathbb{M}_{n}(\mathbb{C})$ be positive. Then

$$
2 s_{j}(M+N) \leqslant\|A+B\|^{1 / 2} s_{j}((X+Y) \oplus(X+Y))
$$

for $j=1,2, \ldots, 2$, where $M=A^{1 / 2} X^{1 / 2} Y^{1 / 2} A^{1 / 2}, N=B^{1 / 2} X^{1 / 2} Y^{1 / 2} B^{1 / 2}$. Letting $Y=X$, we give

$$
s_{j}\left(A^{1 / 2} X A^{1 / 2}+B^{1 / 2} X B^{1 / 2}\right) \leqslant\|A+B\|^{1 / 2} s_{j}(X \oplus X)
$$

for $\mathrm{j}=1,2, \ldots, 2$. Letting $\mathrm{X}=\mathrm{I}$, we give

$$
s_{j}(A+B) \leqslant\|A+B\|^{1 / 2}
$$

for $j=1,2, \ldots, n$.

Proof. Letting $A_{1}=B_{1}=A^{1 / 2}, A_{2}=B_{2}=B^{1 / 2}, X_{1}=X_{2}=X^{1 / 2}, Y_{1}=Y_{2}=Y^{1 / 2}$ and $f(t)=t$ in inequality (2.7), we give inequality (2.10).

Remark 2.16. It should be noted that Inequality (2.10) is sharper than inequality (1.4) when $A=B=I$. To see this, note that $\sqrt{2}_{j}((X+Y) \oplus(X+Y)) \leqslant 2 s_{j}((X+Y) \oplus(X+Y))$.

Corollary 2.17. Let $A, B, X_{1}, X_{2}, Y_{1}, Y_{2} \in \mathbb{M}_{\mathfrak{n}}(\mathbb{C})$. Then

$$
2 s_{j}\left(A X_{1} Y_{1}^{*} A^{*}-B X_{2} Y_{2}^{*} B^{*}\right) \leqslant\left\|A A^{*}+B B^{*}\right\|^{1 / 2} s_{j}(L)
$$

for $j=1,2, \ldots, n$, where

$$
L=\left(X_{1}^{*} X_{1}+Y_{1}^{*} Y_{1}\right) \oplus\left(X_{2}^{*} X_{2}+Y_{2}^{*} Y_{2}\right) .
$$

Proof. In inequality (2.7), letting $A_{1}=B_{1}=A, A_{2}=-B_{2}=B$, and $f(t)=t$, we give inequality (2.11).

Remark 2.18. Letting $X_{2}=Y_{2}=B=0$ in inequality (2.11), we obtain

$$
2 s_{j}\left(A X_{1} Y_{1}^{*} A^{*}\right) \leqslant\left\|A A^{*}\right\|^{1 / 2} s_{j}\left(X_{1}^{*} X_{1}+Y_{1}^{*} Y_{1}\right) .
$$

Inequality (1.1) is a special case of inequality (2.12) for $j=1,2, \ldots, n$.

Remark 2.19. Substituting $A=I$ in inequality (2.12), we obtain inequality (1.1).

The following result follows from inequality (2.11).

Corollary 2.20. Let $A, B, X \in \mathbb{M}_{\mathfrak{n}}(\mathbb{C})$ where $\max \{\|A\|,\|B\|\} \leqslant \frac{1}{2}$ and $X$ is positive. Then

$$
s_{j}\left(A X B^{*}\right) \leqslant \sqrt{2}(\max \{\|A\|,\|B\|\}) s_{j}(X)
$$

for $j=1,2, \ldots, n$.

Proof. In inequality (2.11), let $C=\left[\begin{array}{c}A \\ B\end{array}\right], D=\left[\begin{array}{c}A \\ -B\end{array}\right]$, and $X_{1}=X_{2}=Y_{1}=Y_{2}=X^{1 / 2}$. Then

$$
\mathrm{CX}_{1} \mathrm{Y}_{1}^{*} \mathrm{C}^{*}-\mathrm{DX}_{2} \mathrm{Y}_{2}^{*} \mathrm{D}^{*}=\left[\begin{array}{cc}
0 & 2 \mathrm{AX} \mathrm{B}^{*} \\
2 \mathrm{BX} \mathrm{A}^{*} & 0
\end{array}\right]
$$

and

$$
\mathrm{CC}^{*}+\mathrm{DD}^{*}=2\left[\begin{array}{cc}
\mathrm{AA}^{*} & 0 \\
0 & \mathrm{BB}^{*}
\end{array}\right]
$$

Now, applying inequality (2.11), leads to

$$
2 s_{j}\left(\left[\begin{array}{cc}
0 & \mathrm{AXB}^{*} \\
\mathrm{BXA}^{*} & 0
\end{array}\right]\right) \leqslant \sqrt{2}\left\|\left[\begin{array}{cc}
\mathrm{A} A^{*} & 0 \\
0 & \mathrm{BB}^{*}
\end{array}\right]\right\|^{1 / 2} s_{j}(2 \mathrm{X} \oplus 2 \mathrm{X})
$$


This is equivalent to saying that

$$
\begin{aligned}
s_{j}\left(A X B^{*}\right) & \leqslant \sqrt{2}\left(\max \left\{\left\|A A^{*}\right\|,\left\|B B^{*}\right\|\right\}\right)^{1 / 2} s_{j}(X) \\
& =\sqrt{2}(\max \{\|A\|,\|B\|\}) s_{j}(X) \\
\text { (Since }\|A\|^{2} & \left.=\left\|A^{*} A\right\|=\left\|A A^{*}\right\|\right)
\end{aligned}
$$

for $j=1,2, \ldots, n$. Inequality (2.13) has thus been substantiated.

Remark 2.21. Letting $B=A$ in inequality (2.13), we give

$$
s_{j}\left(A X A^{*}\right) \leqslant \sqrt{2}\|A\| s_{j}(X)
$$

for $j=1,2, \ldots, n$.

Remark 2.22. It should be noted that inequality (2.13) is sharper than inequality (2.1) if $\min \{\|A\|,\|B\|\}>$ $\sqrt{2}$ and inequality (2.14) is sharper than inequality (2.1) if $\|\mathrm{B}\|>\sqrt{2}$.

\section{Acknowledgment}

The author is grateful to the referee for his valuable comments and suggestions, and he is gratitude to University of Petra for its support.

\section{References}

[1] T. Ando, Majorizations and inequalities in matrix theory, Linear Algebra Appl., 199 (1994), 17-67. 1, 2

[2] W. Audeh, Singular value inequalities and applications, Positivity, (2020), 10 pages. 1

[3] W. Audeh, Generalizations for singular value and arithmetic-geometric mean inequalities of operators, J. Math. Anal. Appl., 489 (2020), 8 pages. 1, 2

[4] W. Audeh, Generalizations for singular value inequalities of operators, Adv. Oper. Theory, 5 (2020), 371-381.

[5] W. Audeh, Some generalizations for singular value inequalities of compact operators, Adv. Oper. Theory, 6 (2021), 10 pages.

[6] W. Audeh, F. Kittaneh, Singular value inequalities for compact operators, Linear Algebra Appl., 437 (2012), $2516-2522$. 1

[7] J. S. Aujla, F. C. Silva, Weak majorization inequalities and convex functions, Linear Algebra Appl., 369 (2003), $217-233$. 2

[8] R. Bhatia, Matrix Analysis, Springer, New York, (1997). 1

[9] R. Bhatia, F. Kittaneh, On the singular values of a product of operators, SIAM J. Matrix Anal. Appl., 11 (1990), $272-277$. 1, 2

[10] I. C. Gohberg, M. G. Kreĭn, Introduction to the Theory of Linear Nonselfadjoint Operators, Amer. Math. Soc., Providence, R. I., (1969).

[11] O. Hirzallah, Inequalities for sums and products of operators, Linear Algebra Appl. 407 (2005), 32-42. 1

[12] R. A. Horn, C. R. Johnson, Topics in in Matrix Analysis, Cambridge University Press, (1991). 1 